\title{
What are the evolutionary constraints on larval growth in a trophically transmitted parasite?
}

\author{
Daniel P. Benesh
}

Received: 6 July 2009/ Accepted: 29 October 2009/Published online: 18 November 2009

(c) The Author(s) 2009. This article is published with open access at Springerlink.com

\begin{abstract}
For organisms with a complex life cycle, a large larval size is generally beneficial, but it may come at the expense of prolonged development. Individuals that grow fast may avoid this tradeoff and switch habitats at both a larger size and younger age. A fast growth rate itself can be costly, however, as it requires greater resource intake. For parasites, fast larval growth is assumed to increase the likelihood of host death before transmission to the next host occurs. Using the tapeworm Schistocephalus solidus in its copepod first intermediate host, I investigated potential constraints in the parasite's larval life history. Fast-growing parasites developed infectivity earlier, indicating there is no functional tradeoff between size and developmental time. There was significant growth variation among full-sib worm families, but fast-growing sibships were not characterized by lower host survival or more predation-risky host behavior. Parental investment also had little effect on larval growth rates. The commonly assumed constraints on larval growth and development were not observed in this system, so it remains unclear what prevents worms from exploiting their intermediate hosts more aggressively.
\end{abstract}

Communicated by Steven Kohler.

Electronic supplementary material The online version of this article (doi:10.1007/s00442-009-1507-6) contains supplementary material, which is available to authorized users.

D. P. Benesh ( $\square)$

Department of Evolutionary Ecology,

Max-Planck-Institute for Evolutionary Biology,

August-Thienemann-Strasse 2, 24306 Plön, Germany

e-mail: benesh@evolbio.mpg.de
Keywords Development of infectivity · Host-phenotype manipulation · Maternal effects . Quantitative genetics · Virulence

\section{Introduction}

Organisms with complex life cycles divide their lives into distinct phases, spending their larval and adult lives in different habitats. Larval size is often positively related to fitness, as it may increase survival and fecundity in the adult habitat via e.g., higher competitiveness and/or shorter adult developmental time (e.g., Scott 1994; De Block and Stoks 2005). However, all else being equal, it takes longer to grow to a large size, which increases the risk of dying before moving into the next habitat. This tradeoff between size and developmental time is integral to many life cycle models, and it presumably affects how life cycles are compartmentalized (Werner 1986; Rowe and Ludwig 1991; Stearns 1992; Berrigan and Koella 1994; Abrams et al. 1996; Day and Rowe 2002; Iwasa and Wada 2006).

Growth rate determines the relationship between size and age, and when it varies size-age tradeoffs can be masked. Fast growers may be able to switch habitats at both a larger size and a younger age (see Roff 2000 for examples with regard to size at maturity). Given this clear advantage, selection should increase growth rates. However, rapid growth generally entails costs (Reznick et al. 2000; Gotthard 2001; Angilletta et al. 2003). Fast growth may necessitate high energy intake, additional foraging effort, and thus increased predation risk (Lima 1998), or it may reduce resistance to starvation and other environmental stressors (Arendt 1997; Metcalfe and Monaghan 2001). Maturation may also constrain growth if cell differentiation restricts growth potential (Arendt 1997; Arendt 
2000). In this case, fast growth comes at the cost of prolonged development, although a larger ultimate size is likely reached. Because growth rate is intimately related to development and body size, it plays an important role in determining optimal life history strategies (Abrams et al. 1996; Berner and Blanckenhorn 2007).

Numerous parasites have complex life cycles in which they switch hosts one or more times, often via trophic interactions (Parker et al. 2003a; Poulin 2007). For many parasites, there is likely directional selection for a large transitional size, as it may increase infection success (Rosen and Dick 1983; Steinauer and Nickol 2003) or adult fecundity (Fredensborg and Poulin 2005). However, fast growth and/or a large size is usually assumed to carry an additional cost for larval parasites: increased virulence (Parker et al. 2003b; Ball et al. 2008). That is, rapid parasite growth potentially reduces host survival and thus parasite transmission probability. Increased virulence may not always play a role in limiting larval parasite growth, though (see e.g., Uznanski and Nickol 1980; Benesh and Valtonen 2007).

I examined potential constraints in the larval life history of the tapeworm Schistocephalus solidus. Copepods are the first intermediate host of this species. Parasites that grow larger in copepods induce a lower innate immune response in sticklebacks, the next host, suggesting that a large transitional size is advantageous (Hammerschmidt and Kurtz 2005a). There is between-clutch variation in larval growth (Hammerschmidt and Kurtz 2005a), but it is not known if this leads to switching hosts at a larger size or a younger age. Reduced host survival might not be an important growth cost in this system. Two studies found that copepod survival was unaffected by the number of parasites harbored (Wedekind 1997; van der Veen and Kurtz 2002), even though the burden on the host seems to increase with infection intensity (Wedekind et al. 2000; Michaud et al. 2006). However, Michaud et al. (2006) observed higher copepod mortality in three-worm infections relative to single and double infections. It is not clear if a reduction in host survival at high infection intensities is relevant for natural parasite populations, though, because tapeworms typically infect copepods at very low rates, e.g., Zander et al. (1994), Rusinek et al. (1996), Dörücü (1999) and Hanzelová et al. (2002) all found single-worm infections to be the norm in various cestode-copepod systems. If increased host mortality is an important cost of excessive growth, it should also be observed at more natural infection levels.

Increased mortality with parasite growth could also arise indirectly via changes in host behavior. Infection alters copepod behavior, but the changes depend on parasite development. During the parasite's major growth phase and before becoming infective, copepods seem to have enhanced anti-predator behavior (i.e., lower activity and longer recovery times after disturbance; Hammerschmidt et al. 2009).
After parasites reach infectivity, infected copepods exhibit reduced anti-predator behavior (Hammerschmidt et al. 2009), which likely increases parasite transmission success (Wedekind and Milinski 1996; Jakobsen and Wedekind 1998). During the pre-infectivity period, rapid parasite growth presumably necessitates higher host energy intake. If increased copepod foraging effort ensues, then parasites may be paying for their growth with a higher predation risk, similar to many free-living organisms (Lima 1998). Additionally, if manipulation of host behavior involves energetic costs for parasites, e.g., the production of modulator molecules, then resources may be diverted from growth (Poulin 1994). Hypotheses about the costs of parasite growth (i.e., lower host survival, reduced behavioral manipulation) rely on the assumption that host resources are exploited by parasites. However, tapeworm eggs are provisioned with maternal glycogen and lipids (Swiderski and Xylander 2000), so early parasite development could be energetically independent of host resources. Such maternal effects could eliminate some assumed constraints on larval parasite life history.

I investigated potential tradeoffs in the larval life history of $S$. solidus from both a phenotypic and a genetic perspective. I tested three questions. First, how are larval growth and development related and does growth rate have a masking effect on a tradeoff between size and age at infectivity? Second, what are the costs (lower host survival, reduced host manipulation) associated with increased larval growth? Third, does parental investment affect larval performance?

\section{Materials and methods}

\section{Study system}

Schistocephalus solidus is a simultaneous hermaphrodite with a three-host life cycle. Adult worms live in the intestine of fish-eating birds, where they mate and produce eggs. The eggs are released into the environment with the host feces. Free-swimming, ciliated larvae called coracidia hatch from the eggs and are eaten by freshwater copepods. Parasites penetrate through the copepod's intestine and develop in the body cavity. Transmission to the second intermediate host, three-spined sticklebacks (Gasterosteus aculeatus), occurs when an infected copepod is eaten. Parasites grow for several weeks in sticklebacks before they are able to infect birds.

Infected sticklebacks were collected from Skogseidvatnet, Norway $\left(60^{\circ} 13^{\prime} \mathrm{N} 5^{\circ} 53^{\prime} \mathrm{E}\right)$ with minnow traps and seine nets in 2006. Worms were dissected from the fish, weighed, and then bred in pairs. Pairs were formed assortatively by size in order to promote outcrossing (Lüscher and Milinski 2003), and then placed into an in vitro breeding system that 
simulates a bird gut [developed by Smyth (1946) and modified by Wedekind (1997)]. The average size asymmetry in worm pairs (weight of heavier worm divided by that of the lighter worm) was 1.08, a level at which selfing rates are likely low (Lüscher and Milinski 2003). Each worm pair is hereafter referred to as a "parasite sibship". In this experiment, 12 parasite sibships were used. Eggs were collected and stored at $4{ }^{\circ} \mathrm{C}$ until needed. To induce hatching, eggs were incubated at $20^{\circ} \mathrm{C}$ for 3 weeks in the dark and then exposed to light 1 day before use.

\section{Experimental infection and copepod maintenance}

For the infection, I used a laboratory culture of Macrocyclops albidus [culture maintenance described by van der Veen and Kurtz (2002)]. The lab population was started with about 80 individuals collected from the Kremper $\mathrm{Au}$, a small creek in northern Germany $\left(54^{\circ} 6^{\prime} \mathrm{N} 10^{\circ} 47^{\prime} \mathrm{E}\right)$. The natural copepod hosts of $S$. solidus are not known, but as I infected copepods collected in Germany with Norwegian worms, local (mal)adaptation of host or parasite might have affected the results. However, this seems unlikely for several reasons. First, infection rates of copepods with cestode larvae are usually extremely low (Zander et al. 1994; Rusinek et al. 1996; Dörücü 1999; Hanzelová et al. 2002), suggesting that selection for specialized host resistance is weak. Second, M. albidus is common in the lake from which S. solidus was sampled (P. Jakobsen, personal communication), so it is presumably not an unusual host species for the studied parasite population. Third, a number of copepod species are suitable hosts under experimental conditions (Orr and Hopkins 1969), indicating that the host specificity of the parasite is low. Fourth, a recent microsatellite study of the closely related tapeworm Ligula intestinalis, which has a very similar life cycle to $S$. solidus (i.e., copepod-fishpiscivorous bird), found considerable gene flow between European populations (Štefka et al. 2009). Assuming a similar genetic structure in $S$. solidus, gene flow between populations would probably inhibit adaptation to local copepods. Finally, in a separate experiment, adult male copepods from Germany were infected with two German and two Norwegian parasite sibships. Thirteen days postexposure (PE) Norwegian worms $\left(n=33,24,475 \mu \mathrm{m}^{2} \pm\right.$ $3,873 \mathrm{SD}$ ), were on average slightly bigger than German worms $\left(n=34,21,289 \mu \mathrm{m}^{2} \pm 2,886 \mathrm{SD} ;\right.$ D. P. Benesh and M. Kalbe, unpublished data). As Norwegian worms seem to be somewhat more aggressive, growth costs might be easier to detect in this population.

As I was interested in parasite phenotypes, I attempted to minimize host-related variation. Several inbred copepod families were started with two generations of full-sib matings, and then propagated each generation thereafter with five randomly chosen, egg-bearing females. Thus, genetic diversity within these copepod lines is likely very low. Two families were used in the experiment, and these copepods were from the seventh and eighth generation after the families' founding. Several tanks $(\sim 51)$ were set up that contained ten egg-bearing females from the same family. After 4 weeks, the offspring of these females were used in the infection. Only adult male copepods were taken to eliminate any variation due to copepod stage, sex, and growth (adults do not molt further).

Male copepods were isolated individually in the wells of a 24-well microtitre plate, and starved for 1 day before the exposure. Each copepod was exposed to a single coracidium from one of the 12 parasite sibships. Initially, 1,322 copepods were exposed, with 118 copepods serving as unexposed controls. However, infection levels were low for several sibships, so a second round of the experiment was conducted, with an additional 880 exposed and 80 control copepods. During the experiment, copepods were kept at $18^{\circ} \mathrm{C}$ with a 18:6-h light:dark cycle, and they were fed 11 freshly hatched nauplii of Artemia salina each week. Preliminary experiments suggested that this is slightly below an ad libitum food supply for copepods.

\section{Parasite growth and development}

Starting 7 days PE, copepods were microscopically checked for the presence of tapeworm larvae (Wedekind et al. 2000). Checking the copepods took several days, so the infection status of all copepods was first established at 10 days PE. At 11 days PE, a random subsample of the infected copepods was dissected $(n=215)$. Parasite growth and development were checked at 11 days PE because: (1) between 0 and 11 days $\mathrm{PE}$ growth is approximately linear (Michaud et al. 2006), so size at day 11 gives an indication of growth rate; and (2) there is variation in parasite development at this time (Benesh 2009). Infected copepods were photographed and their length measured from the eye to the end of the cephalothorax (van der Veen and Kurtz 2002). Copepods were then anesthetized with a drop of carbonated water and worms were teased out using fine needles. Live parasites were photographed 2 or 3 times, and worm size (area) was measured using the freeware Image J 1.38x (W. S. Rasband, NIH, Bethesda, Md., http://rsb.info.nih.gov/ij/, 1997 2008). Size measurements on the same worms were highly repeatable between photographs (intra-class correlation between repeated measures, $r=0.942, F_{214,215}=33.59$, $P<0.0001$ ), so these values were simply averaged.

Two developmental traits were assessed: cercomere presence and the area of calcareous corpuscles. The functions of these traits are poorly understood, but their development is well correlated with the attainment of infectivity to the next host (Smyth and McManus 1989). The cercomere is 
a circular structure that forms from the posterior end of larvae. Because it is lost shortly after infection of the next host (e.g., Hammerschmidt and Kurtz 2007), it has often been considered a vestigial organ (Smyth and McManus 1989). Calcareous corpuscles are conspicuous structures composed of organic and inorganic components. They appear and increase in number as worms reach infectivity (Benesh 2009). Hypothesized functions include buffering acid in the next host's stomach, acting as a reservoir of trace nutrients, and suppressing host immunity (Smyth and McManus 1989). To quantify the area of corpuscles, worms were killed with a drop of 5\% formalin and then pressed under a cover slip in $10 \mu \mathrm{l}$ of water. The flattened worms were photographed and total corpuscle area was estimated using Image $\mathrm{J}$.

Infected copepods that were not dissected 11 days PE were used to assess survival, post-infectivity behavioral manipulation, and parasite growth trajectory $(n=204)$. At 21 days $\mathrm{PE}$, all remaining infected copepods were dissected $(n=132)$ and worm size was measured. By this time, parasites are approaching an asymptotic size (Michaud et al. 2006). Only survival 11-21 days PE was assessed, because infection status was established 10 days PE. Two previous studies found copepod survival before 11 days PE to be unaffected by parasite burden (Wedekind 1997; van der Veen and Kurtz 2002), but the one study that followed copepods longer found that hosts harboring three worms have reduced viability (Michaud et al. 2006). Here, using single-worm infections, I examined whether host survival is related to parasite growth after parasites have become infective. Survival was treated as a discrete character (i.e., alive or dead at 21 days PE), because copepods were not checked at regular intervals.

\section{Behavioral observations}

Copepod behavior was recorded 3 times during the experiment: 6, 15, and 20 days PE. These dates reflect different stages in the parasite's development: before infectivity, shortly after reaching infectivity, and well after becoming infective (Hammerschmidt et al. 2009). The apparatus for recording copepod behavior was detailed elsewhere (Hammerschmidt et al. 2009). Briefly, a 24-well plate with copepods was held a few millimeters above the ground before being dropped. The impact "frightened" copepods and simulated a standardized predator attack. After this shock, copepods were recorded for 6 min with a digital camera (Panasonic WV-BP550). Two aspects of behavior were quantified: latence time and activity. Latence time was defined as the time between the initial shock and a copepod's first movement, excluding the initial flight response. Activity was the amount of time copepods spent moving during the last minute of recording (i.e., between 5 and $6 \mathrm{~min}$ after the initial shock). Copepod activity is relevant to predation risk because active copepods attract the attention of sticklebacks and are attacked (Wedekind and Milinski 1996). Behavior at 6 days PE was evaluated for all infected copepods that survived at least 10 days PE $(n=440)$, and behavior 15 and 20 days PE was evaluated for those that survived until 21 days PE $(n=132)$. Subsamples of unexposed controls ( $n=139$ for 6 days PE; $n=56$ for 15 and 20 days PE) and exposed, uninfected copepods $(n=178$ for 6 days PE; $n=52$ for 15 and 20 days PE) were also assessed.

\section{Parental effects}

Average parental weight, parental size discrepancy (predicts outcrossing rate; see Lüscher and Milinski 2003), egg size, coracidia survival, and hatching rate were recorded for each of the 12 clutches. These traits may affect larval life history, though they are better considered as characteristics of parental worms than of offspring. S. solidus is a simultaneous hermaphrodite, and both worms in a pair produce eggs. As mother worms cannot be identified, I refer to these potential effects as "parental" rather than "maternal". From each clutch, the area $\left(\mu \mathrm{m}^{2}\right)$ of 40 randomly selected eggs was measured using Image J. To assess coracidia survival, freshly hatched larvae (48 per sibship) were placed individually in the wells of 96-well microtitre plates $(150 \mu \mathrm{l}$ per well). After $12 \mathrm{~h}$, the survival of the coracidia was checked. Hatching success was estimated by counting the eggs with open opercula in a random sample of 100 eggs. Counts were conducted 3 months after eggs were initially exposed to light, so as to allow sufficient time for all viable larvae to hatch.

Data analyses

Of the exposed copepods, 279 (19.7\%) did not survive until being checked for infection; this mortality was evenly distributed among parasite sibships $\left(\chi_{11}^{2}=12.56\right.$, $P=0.323)$. These copepods were removed from all analyses. For each parasite trait, a generalized linear model was constructed in SPSS version 16.0 (SPSS, Chicago, Ill.). Continuous variables (size, corpuscle volume) were modeled as normally distributed with an identity link function while binary data (infectivity, cercomere presence, survival) were modeled with a binomial distribution and logit link function. Raw behavioral data were not normally distributed, but log-transformed data were roughly normal. Thus, behavioral traits were modeled as normally distributed but with a log transformation as the link function. All models included parasite sibship, copepod family, and infection round as factors. When possible, copepod size was also included as a covariate. I began with fully saturated models and then sequentially removed non-significant interaction terms so as to produce the most 
parsimonious models (judged by Akaike's information criterion). Only relevant results are presented in the text; full models are given in the supplementary material. Using these models, sibship averages for each trait were estimated for use in quantitative genetic analyses.

The experiment was a full-sib design, which cannot account for dominance, maternal, or common environment effects (Roff 1997), so trait heritabilities were estimated in the broad sense. However, as parasites develop in separate hosts, common environment effects are negligible. Presumably, the majority of the eggs within a clutch were outcrossed, i.e., fullsibs, but some might have been selfed. This could either increase or decrease heritability estimates, depending on the similarity of outcrossed and selfed offspring. Variance components were computed with restricted maximum likelihood, as implemented with the VARCOMP function in SPSS. Heritabilities and their standard errors were calculated according to the formulas for continuous and threshold (discrete) traits given by Roff (1997, pp 41-42 and pp 51-52).

Several traits could not be measured within individuals (e.g., size 11 and 21 days PE), so, to assess genetic correlations between traits, the family mean approach proposed by Via (1984) was used. Essentially, the Pearson correlation between family averages for two traits is taken as an approximation of the genetic correlation. This estimate includes a portion of the within family variance, and should thus be treated as an upper boundary for the genetic correlation (Roff 1997). Moreover, Roff and Preziosi (1994) pointed out that estimates with this method can be biased upwards when family sizes are small. For all traits, average family sizes were $>10$ (minimal mean family size was 10.8 for behavior 15 and 20 days PE as well as size 21 days PE). At this level, substantial bias only occurs when phenotypic and genetic correlations are highly divergent (Roff and Preziosi 1994). The family mean approach was also used to examine potential parental effects on larval performance. Clutch averages were computed for parental traits and compared with averages for larval traits. In total, correlations between 17 traits were assessed, so the probability of false positives was relatively high. Therefore, individual correlations are not emphasized. Instead, particular attention is given to suites of significantly intercorrelated traits, which are far less likely to arise by chance (Moran 2003).

\section{Results}

Infectivity, growth, and development

Of the exposed copepods, $25.7 \%$ were infected. Infectivity, size at 11 and 21 days PE, and calcareous corpuscle volume all exhibited significant between-sibship variation (Table 1), but cercomere presence did not (Wald $\chi_{11}^{2}=15.8$,
Table 1 Broad-sense heritability estimates for larval traits in Schistocephalus solidus

\begin{tabular}{llr}
\hline Trait & Heritability (SE) & \multicolumn{1}{c}{$n$} \\
\hline Infectivity & $\mathbf{0 . 1 7}(\mathbf{0 . 0 8})^{\mathrm{a}}$ & $\mathbf{1 , 9 0 7}$ \\
Larval size 11 days PE & $\mathbf{0 . 3 3}(\mathbf{0 . 1 5})$ & $\mathbf{2 1 5}$ \\
Cercomere presence & $0.29(0.21)$ & 215 \\
Calcareous corpuscle volume & $\mathbf{0 . 3 2}(\mathbf{0 . 1 5})$ & $\mathbf{2 1 5}$ \\
Survival between 11 and 21 days PE & $0.00(0.12)$ & 204 \\
Larval size 21 days PE & $\mathbf{0 . 5 2}(\mathbf{0 . 2 2})$ & $\mathbf{1 3 2}$ \\
Host activity 6 days PE & $\mathbf{0 . 1 5}(\mathbf{0 . 0 8})$ & $\mathbf{4 4 0}$ \\
Host latence time 6 days PE & $0.00(0.02)$ & 440 \\
Host activity 15 days PE & $0.03(0.09)$ & 132 \\
Host latence time 15 days PE & $0.00(0.08)$ & 132 \\
Host activity 20 days PE & $0.00(0.08)$ & 132 \\
Host latence time 20 days PE & $0.00(0.08)$ & 132 \\
\hline
\end{tabular}

$P E$ Post-exposure

${ }^{a}$ Traits with significant between-sibship variation, as determined by generalized linear models, are indicated in bold

$P=0.149$ ). However, $82.8 \%$ of worms had developed a cercomere by 11 days $\mathrm{PE}$, so there was relatively little variation with which to detect between-sibship differences.

Worms with a cercomere were larger than those without one (Mann-Whitney $U$-test, $Z=7.94, P<0.0001$ ), and there was a positive correlation between size and corpuscle volume (Pearson correlation, $r=0.739, F_{1,213}=255.9$, $P<0.0001)$. Thus, large worms were further developed at 11 days PE (Fig. 1). The sibship averages for size at 11 days PE and both developmental traits were positively correlated (between size and cercomere presence $r=0.77$ and between size and corpuscle area $r=0.84$; Table 2), suggesting the connection between growth and

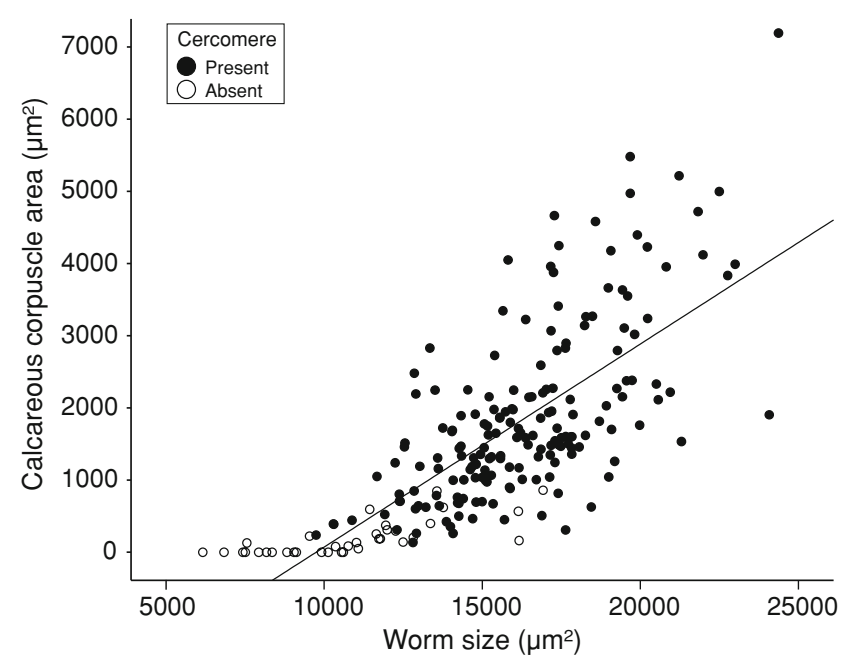

Fig. 1 Relationship between size and calcareous corpuscle development in the parasitic tapeworm Schistocephalus solidus 11 days postinfection. Worms with a cercomere are represented by solid circles, worms without a cercomere are represented by open circles 


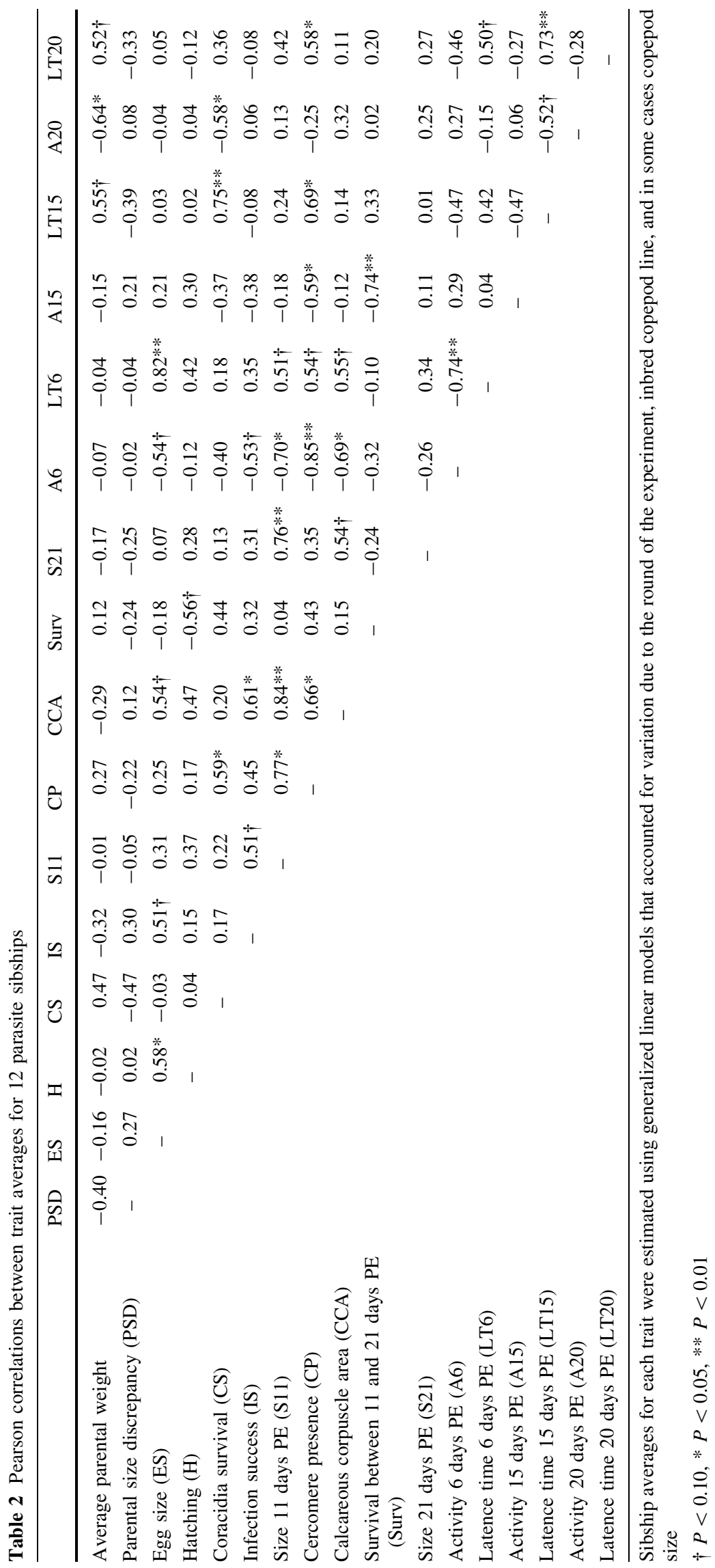


development has a large genetic component. There was also a strong positive correlation between size 11 and 21 days PE $(r=0.76$; Table 2$)$, indicating that faster growing worms tend to reach a larger ultimate size.

Growth costs?

\section{Survival}

Between 11 and 21 days PE, the survival of unexposed controls and exposed, uninfected copepods did not differ, so these groups were pooled $\left(\chi^{2}\right.$-test on proportion of copepods surviving, $\left.\chi_{1}^{2}=2.14, P=0.144\right)$. The survival of these pooled, uninfected copepods was not significantly different from that of infected copepods (percentages of uninfected and infected copepods alive 21 days PE, 62.2 and $64.7 \%$, respectively; $\chi^{2}$-test, $\chi_{1}^{2}=0.49, P=0.482$ ). There was no significant between-sibship variation in host survival (Wald $\left.\chi_{11}^{2}=8.57, P=0.661\right)$, and parasite growth and host survival were unrelated at the sibship level ( $r=0.04$; Table 2).

\section{Behavior}

For all behavioral measures, unexposed controls and exposed, uninfected copepods did not significantly differ (Mann-Whitney $U$-tests, all $P \geq 0.232$ ), so these groups were pooled. Throughout the experiment, infected copepods had longer latence times and were less active than uninfected copepods (Mann-Whitney $U$-tests for both latence time and activity, all $P<0.002$; Fig. 2).

There was significant variation between parasite sibships for activity 6 days PE (Wald $\chi_{11}^{2}=48.32, P<0.0001$ ), but not for the other behavioral traits (Table 1). At the level of individual parasites, growth during the pre-infectivity period did not appear to affect host behavior (Spearman correlations between behavior 6 days PE and parasite size 11 days PE, $n=215$; latence time, $\rho=-0.12, P=0.08$; activity, $\rho=0.03, P=0.64$; Fig. 3). However, at the sibship level, fast parasite growth was associated with lower host activity 6 days PE ( $r=-0.70$; Table 2; Fig. 3), and perhaps longer latence times $(r=0.51$; Table 2$)$. Early developing sibships also tended to have lower host activity and longer latence times 6 days PE (Table 2). Host behavior 15 and 20 days PE was unrelated to parasite size both at the level of individuals (Spearman correlations between behavior 15 and 20 days PE and parasite size 21 days $\mathrm{PE}, n=132$, all $\rho<0.10$, $P>0.27$ ) and at the sibship level (Table 2).

\section{Parental effects}

Parental weight, parental size discrepancy, and hatching rate were not correlated with larval performance (Table 2). Egg size and coracidia survival were weakly associated with
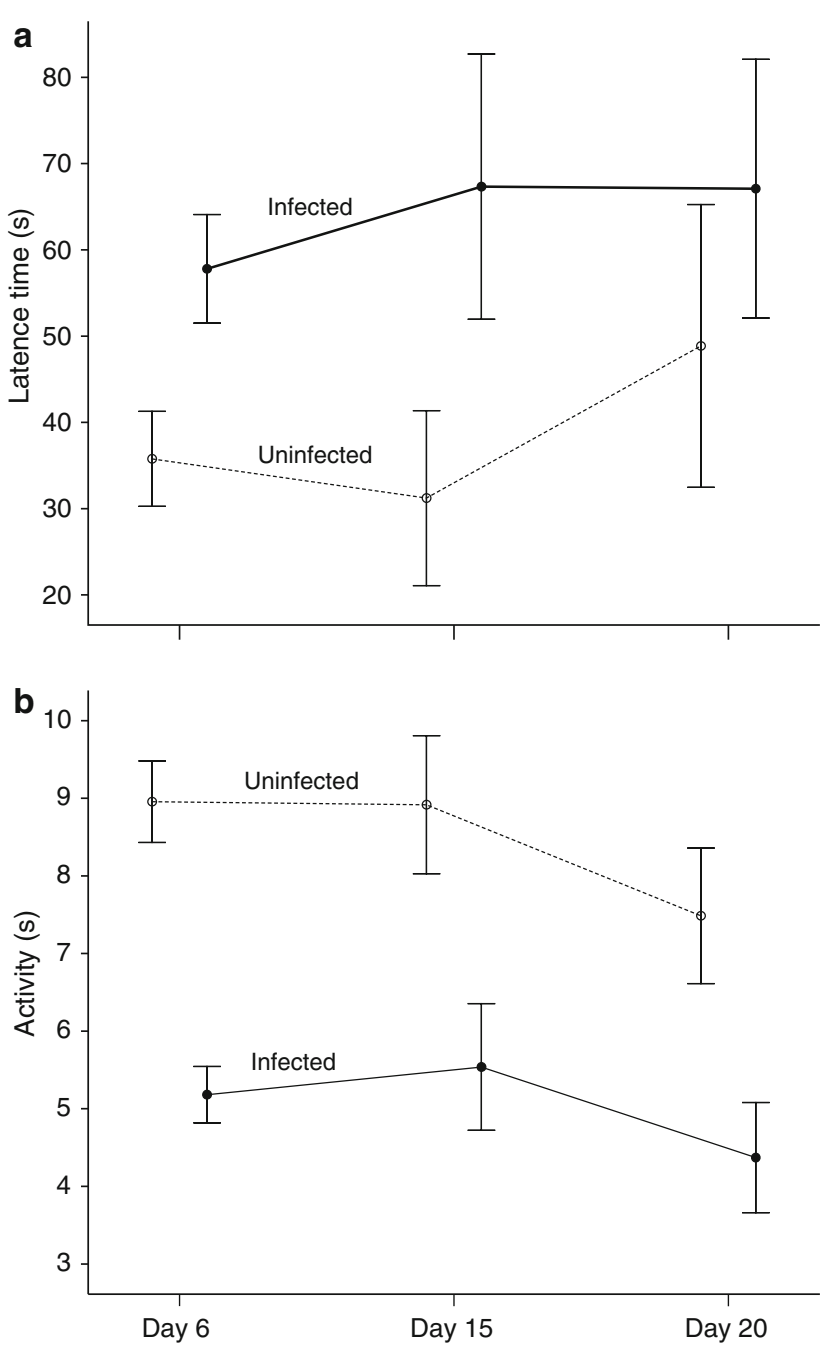

Fig. 2 a Latence time (number of seconds between an initial shock and a copepod's first movement) and $\mathbf{b}$ activity (number of seconds copepods spent moving during $1 \mathrm{~min}$ of observation) of uninfected and infected copepods at 6, 15, and 20 days post-exposure (mean $\pm 95 \%$ confidence intervals)

some larval traits. Larger eggs were associated with better infection success, greater corpuscle development, and more altered host behavior 6 days PE, whereas coracidia survival was associated with cercomere development and postinfectivity host behavior (Table 2). However, these trends were not strong (generally $r<0.6$ ), which suggests parental effects are not major determinants of larval life history.

\section{Discussion}

Larval performance

Parasite size at 11 days PE, development at 11 days PE, and size at 21 days PE formed a suite of positively correlated traits; worms that grew faster, also developed faster, and 


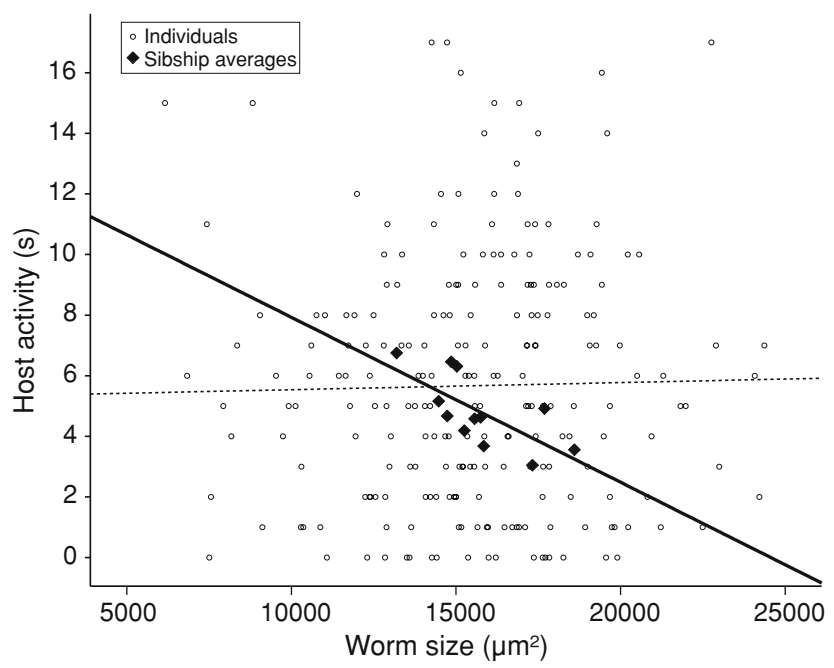

Fig. 3 Relationship between worm size and host activity 11 days post-infection. Both individual data points (open circles, dashed line) and average values for parasite sibships (filled diamonds, solid line) are plotted

reached a larger ultimate size. Under a minimum size, worms had not developed a cercomere or corpuscles 11 days PE (Fig. 1). If a threshold size must be attained before switching hosts, then fast growers may develop infectivity earlier simply because they quickly reach this threshold (Day and Rowe 2002). However, I infected different copepod stages with $S$. solidus, and found that worms grew more slowly in smaller host stages, but this did not notably affect development (Benesh 2009). Many other tapeworm species also seem to be able to develop at a normal pace under very poor larval growth conditions (Benesh 2009). Thus, size thresholds do not seem critical for development and are unlikely to generate the positive genetic correlation between growth and ontogeny. Presumably, the mechanisms underlying this correlation (rates of cell division?) differ from those underlying growth plasticity (changes in cell size?).

Rapid growth in $S$. solidus results in both a larger size and a younger age at infectivity, but it is not obvious that the former is fitness relevant. Hammerschmidt and Kurtz (2005a) observed a similar level of between-sibship variation in larval growth (heritability $=0.29$ vs. 0.33 observed here), and they found it to be related to the innate immune response of sticklebacks, the next host. However, there was not a link between larval size and incontrovertible fitness traits, such as infection success or growth in fish (Hammerschmidt and Kurtz 2005a). Nonetheless, quicker development presumably reduces mortality rate, so rapid growth should be favored unless there is some cost opposing this selection.

Costs of larval growth

Infective worms develop distinct tegumental layers (Hammerschmidt and Kurtz 2005b), and this type of tissue differentiation might restrict growth potential (Arendt 1997, 2000). Fast-growing and early maturing parasite sibships, however, tended to attain a larger size 21 days $\mathrm{PE}$, indicating maturation does not prevent additional growth.

For parasites, fast growth is thought to come at the cost of higher virulence (Ball et al. 2008). Here and in other studies (Wedekind 1997; van der Veen and Kurtz 2002), there was no relationship between parasite burden and host survival. In fact, $S$. solidus could probably grow even faster without risking killing its host. Michaud et al. (2006) observed total worm volume to increase faster in twoworm infections, relative to single infections, but this did not result in higher host mortality. Thus, when alone, worms seem to exploit their host at a submaximal, low-risk level. This appears to be the case for other trophically transmitted parasites as well (Uznanski and Nickol 1980; Benesh and Valtonen 2007).

Growth costs were also not manifested in host behavior. Parasite growth was unrelated to host behavior at 15 and 20 days PE. At 6 days PE, though, faster-growing, early maturing parasite sibships were characterized by lower host activity and longer host latence times. Low activity reduces conspicuousness to sticklebacks (Wedekind and Milinski 1996). Thus, unlike in free-living organisms where fast growth often increases predation risk (Lima 1998), rapid parasite growth appears associated with predation avoidance behaviors in the host, which benefits parasites as long as they are not infective. However, at the individual level, copepods infected with larger parasites did not have more extensively altered behavior 6 days PE. Thus, phenotypic and genetic correlations are divergent, suggesting environmental noise may obscure a genetic relationship between parasite growth and host behavior. This kind of discrepancy can occur when heritabilities are low (Roff 1997), as was the case for behavior, so the negative genetic correlation between growth and host activity 6 days PE should be interpreted cautiously. Additionally, it should be noted that there was weak, but significant variation between parasite sibships in copepod activity 6 days PE, which, to my knowledge, is the first indication that host behavioral modification is genetically variable within a parasite population.

Unlike in previous experiments (Wedekind and Milinski 1996; Hammerschmidt et al. 2009), infected copepods were not more active than uninfected copepods 15 and 20 days PE, i.e., there was not a switch in host behavior as parasites reached infectivity. The reason for this is unknown. Perhaps there are differences between parasite populations in the existence or time course of host behavioral alteration, or post-infectivity manipulation may only occur in specific host strain-parasite strain combinations. 
Parental effects

Maternal provisioning could give some parasites a head start in their larval life. There was variation between clutches in traits like egg size and coracidia survival (see Supplementary Table 3), suggesting differential parental investment, but this had either no or only a weak effect on larval performance. Thus, parental effects seem relatively unimportant in determining larval success.

What constrains larval parasite life history?

Growth variation in larval $S$. solidus appears to mask a sizeage tradeoff, fast larval growth does not clearly affect host viability or host behavior, and residual effects of parental investment appear minimal. Thus, it is unclear what prevents $S$. solidus from evolving to exploit copepods more aggressively. One possibility is that growth costs arise in stressful environments (e.g., Arendt 1997; Gotthard 2001; Metcalfe and Monaghan 2001), such as in small or starved copepods. The growth of $S$. solidus is reduced in small hosts (Wedekind et al. 2000; Benesh 2009), which may either be a simple response to resource availability or a tactic to avoid virulence costs (Parker et al. 2003b). Surprisingly, growth is not strongly reduced in starved copepods (Benesh 2009), which might be risky for parasites. Another alternative hypothesis is that parasites trade off performance in their various hosts, with good larval performers doing less well as adults (Davies et al. 2001; Walker et al. 2006). Hammerschmidt and Kurtz (2005a) did not find a tradeoff between $S$. solidus growth in copepods and parasite performance in sticklebacks, though they suggested that good growers in copepods may be worse at evading the fish's adaptive immune response. Ebenman (1992) argued that selection should break up genetic correlations between traits expressed in very different habitats, i.e., between-host tradeoffs should be eliminated. Larval performance may also depend on host-parasite compatibility. The good growers may have simply been well-suited to exploit the copepods used in the experiment, and they may perform relatively poorly in other host strains or species. However, here and in a separate study (Benesh 2009), larval growth was not influenced by genotype-genotype interactions (i.e., parasite sibship by copepod family). As a range of copepod species are susceptible (Orr and Hopkins 1969), specialization on particular host types seems unlikely in $S$. solidus. Nonetheless, before specialization can be excluded as a determinant of larval growth, experiments with a wider range of host genotypes should be conducted.

Larval growth and the timing of host-switching are important life history traits. Therefore, understanding complex life cycles as adaptive strategies (Parker et al. 2003a) requires knowledge about the evolutionary constraints on larval host exploitation. Here, I emphasize that commonly assumed tradeoffs, such as between size and age at infectivity or between growth and virulence, need not apply to all parasites. The alternative constraints, if any, occurring in the larval life history of trophically transmitted parasites remain to be elucidated.

Acknowledgments Thanks to R. Leipnitz for help throughout the experiment and to M. Kalbe and P. Jakobsen for collecting and breeding the worms. M. Milinski, M. Kalbe, P. Jakobsen, and C. Eizaguirre provided helpful comments on an earlier version of the manuscript. The experiments complied with German law.

Open Access This article is distributed under the terms of the Creative Commons Attribution Noncommercial License which permits any noncommercial use, distribution, and reproduction in any medium, provided the original author(s) and source are credited.

\section{References}

Abrams PA, Leimar O, Nylin S, Wiklund C (1996) The effect of flexible growth rates on optimal sizes and development times. Am Nat 147:381-395

Angilletta MJ, Wilson RS, Navas CA, James RS (2003) Tradeoffs and the evolution of thermal reaction norms. Trends Ecol Evol $18: 234-240$

Arendt JD (1997) Adaptive intrinsic growth rates: an integration across taxa. Q Rev Biol 72:149-177

Arendt JD (2000) Allocation of cells to proliferation vs. differentiation and its consequences for growth and development. J Exp Zool 288:219-234

Ball MA, Parker GA, Chubb JC (2008) The evolution of complex life cycles when parasite mortality is size- or time-dependent. J Theor Biol 253:202-214

Benesh DP (2009) Developmental inflexibility of larval tapeworms in response to resource variation. Int J Parasitol (in press)

Benesh DP, Valtonen ET (2007) Effects of Acanthocephalus lucii (Acanthocephala) on intermediate host survival and growth: implications for exploitation strategies. J Parasitol 93:735-741

Berner D, Blanckenhorn WU (2007) An ontogenetic perspective on the relationship between size and age at maturity. Funct Ecol 21:505-512

Berrigan D, Koella JC (1994) The evolution of reaction norms: simple models for age and size at maturity. J Evol Biol 7:549-566

Davies CM, Webster JP, Woolhouse MEJ (2001) Trade-offs in the evolution of virulence in an indirectly transmitted macroparasite. Proc R Soc Lond B 268:251-257

Day T, Rowe L (2002) Developmental thresholds and evolution of reaction norms for age and size at life-history transitions. Am Nat 159:338-350

De Block M, Stoks R (2005) Fitness effects from egg to reproduction: bridging the life-history transition. Ecology 86:185-197

Dörücü M (1999) Seasonal variation of Pseudophyllidean cestode, Diphyllobothrium spp. infection in Cyclops strenuous abyssorum (Copepoda) in Loch Lomond. Turk J Zool 23:85-91

Ebenman B (1992) Evolution in organisms that change their niches during the life cycle. Am Nat 139:990-1021

Fredensborg BL, Poulin R (2005) Larval helminthes in intermediate hosts: does competition early in life determine the fitness of adult parasites? Int J Parasitol 35:1061-1070 
Gotthard K (2001) Growth strategies of ectothermic animals in temperate environments. In: Atkinson D, Thorndyke M (eds) Environment and animal development. BIOS, Oxford, pp 287304

Hammerschmidt K, Kurtz J (2005a) Evolutionary implications of the adaptation to different immune systems in a parasite with a complex life cycle. Proc R Soc Lond B 272:2511-2518

Hammerschmidt K, Kurtz J (2005b) Surface carbohydrate composition of a tapeworm in its consecutive intermediate hosts: individual variation and fitness consequences. Int $\mathrm{J}$ Parasitol 35:1499-1507

Hammerschmidt K, Kurtz J (2007) Schistocephalus solidus: establishment of tapeworms in sticklebacks-fast food or fast lane? Exp Parasitol 116:142-149

Hammerschmidt K, Koch K, Milinski M, Chubb JC, Parker GA (2009) When to go: optimization of host switching in parasites with complex life cycles. Evolution 63:1976-1986

Hanzelová V, Scholz T, Gerdeaux D, Kuchta R (2002) A comparative study of Eubothrium salvelini and E. crassum (Cestoda: Pseudophyllidea) parasites of Arctic charr and brown trout in alpine lakes. Environ Biol Fishes 64:245-256

Iwasa Y, Wada G (2006) Complex life cycle and body sizes at lifehistory transitions for macroparasites. Evol Ecol Res 8:14271443

Jakobsen PJ, Wedekind C (1998) Copepod reaction to odor stimuli influenced by cestode infection. Behav Ecol 9:414-418

Lima SL (1998) Stress and decision making under the risk of predation: recent developments from behavioral, reproductive, and ecological perspectives. Adv Stud Behav 27:215-290

Lüscher A, Milinski M (2003) Simultaneous hermaphrodites reproducing in pairs self-fertilize some of their eggs: an experimental test of predictions of mixed-mating and Hermaphrodite's Dilemma theory. J Evol Biol 16:1030-1037

Metcalfe NBM, Monaghan P (2001) Compensation for a bad start: grow now, pay later? Trends Ecol Evol 16:254-260

Michaud M, Milinski M, Parker GA, Chubb JC (2006) Competitive growth strategies in intermediate hosts: experimental tests of a parasite life-history model using the cestode, Schistocephalus solidus. Evol Ecol 20:39-57

Moran MD (2003) Arguments for rejecting the sequential Bonferroni in ecological studies. Oikos 100:403-405

Orr TSC, Hopkins CA (1969) Maintenance of Schistocephalus solidus in the laboratory with observations on rate of growth of, and proglottid formation in, the plerocercoid. J Fish Res Bd Can 26:741-752

Parker GA, Chubb JC, Ball MA, Roberts GN (2003a) Evolution of complex life cycles in helminth parasites. Nature 425:480-484

Parker GA, Chubb JC, Roberts GN, Michaud M, Milinski M (2003b) Optimal growth strategies of larval helminths in their intermediate hosts. J Evol Biol 16:47-54

Poulin R (1994) The evolution of parasite manipulation of host behaviour: a theoretical analysis. Parasitology 109:S109-S118

Poulin R (2007) Evolutionary ecology of parasites, 2nd edn. Princeton University Press, Princeton

Reznick D, Nunney L, Tessier A (2000) Big houses, big cars, superfleas, and the costs of reproduction. Trends Ecol Evol $15: 421-425$

Roff DA (1997) Evolutionary quantitative genetics. Chapman and Hall, New York

Roff DA (2000) Trade-offs between growth and reproduction: an analysis of the quantitative genetic evidence. J Evol Biol $13: 434-445$
Roff DA, Preziosi R (1994) The estimation of the genetic correlation: the use of the jackknife. Heredity 73:544-548

Rosen R, Dick TA (1983) Development and infectivity of the procercoid of Triaenophorus crassus Forel and mortality of the first intermediate host. Can J Zool 61:2120-2128

Rowe L, Ludwig D (1991) Size and timing of metamorphosis in complex life cycles: time constraints and variation. Ecology 72:413-427

Rusinek OT, Bakina MP, Nikolskii AV (1996) Natural infection of the calanoid crustacean Epischura baicalensis by procercoids of Proteocephalus sp. in Listvenichnyi Bay, Lake Baikal. J Helminthol 70:237-247

Scott DE (1994) The effect of larval density on adult demographic traits in Ambystoma opacum. Ecology 75:1383-1396

Smyth JD (1946) Studies on tapeworm physiology. I. Cultivation of Schistocephalus solidus in vitro. J Exp Biol 23:47-70

Smyth JD, McManus DP (1989) The physiology and biochemistry of cestodes. Cambridge University Press, Cambridge

Stearns SC (1992) The evolution of life histories. Oxford University Press, Oxford

Štefka J, Hypša V, Scholz T (2009) Interplay of host specificity and biogeography in the population structure of a cosmopolitan endoparasite: microsatellite study of Ligula intestinalis (Cestoda). Mol Ecol 18:1187-1206

Steinauer ML, Nickol BB (2003) Effect of cystacanth body size on adult success. J Parasitol 89:251-254

Swiderski Z, Xylander WER (2000) Vitellocytes and vitellogenesis in cestodes in relation to embryonic development, egg production and life cycle. Int J Parasitol 30:805-817

Uznanski RL, Nickol BB (1980) Parasite population regulation: lethal and sublethal effects of Leptorhynchoides thecatus (Acanthocephala: Rhadinorhynchidae) on Hyalella azteca (Amphipoda). J Parasitol 66:121-126

van der Veen IT, Kurtz J (2002) To avoid or eliminate: cestode infections in copepods. Parasitology 124:465-474

Via S (1984) The quantitative genetics of polyphagy in an insect herbivore. II. Genetic correlations in larval performance within and among host plants. Evolution 38:896-905

Walker SM, Hoey E, Fletcher H, Brennan G, Fairweather I, Trudgett A (2006) Stage-specific differences in fecundity over the lifecycle of two characterized isolates of the liver fluke, Fasciola hepatica. Parasitology 133:209-216

Wedekind C (1997) The infectivity, growth and virulence of the cestode Schistocephalus solidus in its first intermediate host, the copepod Macrocyclops albidus. Parasitology 115:317-324

Wedekind C, Milinski M (1996) Do three-spined sticklebacks avoid consuming copepods, the first intermediate host of Schistocephalus solidus? - an experimental analysis of behavioural resistance. Parasitology 112:371-383

Wedekind C, Christen M, Schärer L, Treichel N (2000) Relative helminth size in crustacean hosts: in vivo determination, and effects of host gender and within-host competition in a copepod infected by a cestode. Aquat Ecol 34:279-285

Werner EE (1986) Amphibian metamorphosis: growth rate, predation risk, and the optimal size at transformation. Am Nat 128:319341

Zander CD, Groenewold S, Strohbach U (1994) Parasite transfer from crustacean to fish hosts in the Lübeck Bight, SW Baltic Sea. Helgol Mar Res 48:89-105 